the mean score was lower among the unintended and unplanned groups (difference in mean score was -4.8 (95\% CI -6.0 to 3.7$)$ and $-2.8(95 \% \mathrm{CI}-3.4$ to -1.51$)$ respectively) and higher in the ART group (3.8 (95\% CI -0.2 to 7.85$)$ ). This equates to a 3.8 month cognitive delay in the unintended group, and to a 3.0 month advantage in the ART group, compared to the planned group. These effects almost entirely disappear once the models are adjusted for sociodemographic factors (parent's income, qualifications, mother's age, whether the baby is her first child and alcohol in pregnancy). Compared to the fertile group, the difference in means becomes -0.9 (95\% CI -1.9 to 0.2$)$ in the unintended group, -0.4 (95\% CI -1.2 to 0.4$)$ in the planned group and $1.2(95 \% \mathrm{CI}-2.0$ to 4.4$)$ in the ART group. Further adjustment for lifecourse mediating factors (birthweight, parent-child relationship, and parental involvement) makes little difference to the final results.

Conclusions We find no evidence that pregnancy planning, subfertility or ART adversely affects children's expressive language ability at 3 years of age. The effects observed in the crude analyses are almost entirely explained by confounding by socio-economic factors, indicating that maternal education and poverty are strong influences on children's cognitive tests in early childhood.

\section{Provision of services 062 ABOLITION OF PRESCRIPTION CO-PAYMENTS IN WALES: AN OBSERVATIONAL STUDY ON DISPENSING RATES}

doi:10.1136/jech.2010.120956.62

${ }^{1} \mathrm{D}$ Cohen, ${ }^{1} \mathrm{~F}$ Alam, ${ }^{2} \mathrm{~F}$ Dunstan, ${ }^{1} \mathrm{~S}$ Myles, ${ }^{3} \mathrm{D}$ Hughes, ${ }^{2} \mathrm{P}$ Routledge. ${ }^{1} \mathrm{Health}$ Economics and Policy Research Unit, University of Glamorgan, Pontypridd, UK; ${ }^{2}$ School of Medicine, Cardiff University, Cardiff, UK; ${ }^{3}$ Centre for Economics and Policy in Health, Bangor University, Bangor, UK

Objective To assess the effects of the abolition in April 2007 of prescription co-payments in Wales on rates of dispensing by general practices.

Design and setting An observational study comparing changes in dispensing in Wales, where co-payments were abolished, with North East England where they continued.

Participants All general practices in Wales and North East England which provided monthly dispensing data continuously between October 2003 and March 2008.

Main outcome measures The main outcome was the change in dispensing of all BNF items per 1000 patients per month between the baseline year before charges were first reduced in Wales and the year following abolition. Similar comparisons were made for the 14 medicines that had previously had most items dispensed subject to charge before abolition. Sales of over-the-counter substitutes were also examined where relevant. A survey of local health boards and primary care trusts examined local initiatives, which might differentially affect dispensing between the two areas.

Results Total monthly dispensing rates (items/1000 patients) increased significantly in both areas but significantly less so in Wales (difference $=-19.7, p=0.024,95 \% \mathrm{CI}-36.7$ to -2.6 ). For the 14 selected medicines, combined dispensing rates increased significantly in both areas but significantly more in Wales (difference=27.51, $\mathrm{p}<0.0001,95 \%$ CI 23.66 to 31.35). There was much variation for individual drugs, but BNF categories tended to show this same trend except for antibiotics, where rates increased in Wales but decreased in NE England. The survey revealed few local initiatives that could explain these differences. Sales of over-the counter substitutes did not explain the changes in dispensing.

Conclusion The Welsh policy was associated with a smaller increase in dispensing of all BNF items than NE England. There was a small increase in dispensing rates relative to NE England for the 14 medicines with the highest number of items dispensed subject to charge before abolition. Although factors besides the co-payment may have influenced these changes, these results suggest that the overall impact of abolition was minimal.

\section{POPULATION AGEING IN SCOTLAND: AN ANALYSIS OF THE IMPLICATIONS FOR HEALTHCARE EXPENDITURE USING THE RENFREW/PAISLEY STUDY}

doi:10.1136/jech.2010.120956.63

${ }^{1} \mathrm{C}$ Geue, ${ }^{2} \mathrm{P}$ Lorgelly, ${ }^{1} \mathrm{~J}$ Lewsey, ${ }^{1} \mathrm{~A}$ Briggs, ${ }^{1} \mathrm{C}$ Hart. ${ }^{1}$ University of Glasgow, Public Health and Health Policy, Division of Community Based Sciences, Glasgow, UK; ${ }^{2}$ Monash University, Centre for Health Economics, Clayton, Australia

Objective It is important for health policy and expenditure projections to fully understand the relationship between age, death and expenditure for health care (HC). Evidence shows that older age groups incur less acute $\mathrm{HC}$ costs than previously anticipated and that time to death (TTD) may be a much stronger indicator for HC expenditure than age alone. Our objective was to undertake research into the relationship between TTD, age and HC expenditure in Scotland.

Methods We use a longitudinal data set (the Renfrew/Paisley study, one of the Midspan studies) covering a period of 35 years, and including baseline survey data linked to subsequent hospital admissions (Scottish Morbidity Records) and death records, to estimate the independent effect that age and TTD have on expenditure for acute inpatient care. We include variables previously omitted in other studies such as deprivation category. This is of special interest in Scotland, with high levels of deprivation in some areas and a poor record of premature deaths. We estimate HC costs in the last 60 quarters before death. We use speciality and hospital specific cost data from the Scottish Cost Book. We estimate HC expenditure conditional on having had a hospitalisation within a particular quarter before death. Due to the skewed nature of our cost variable we employ a generalised linear regression model with a Gamma distribution and a log link and use clustering on patient identifier to control for unobserved within-patient effects.

Results Regression results for age and its quadratic form show that on average older individuals incur higher costs $(p<0.001)$ but at a decreasing rate, which means that for the very old costs are declining. After including a measure of remaining TTD we find that age at death has a non significant association with costs but TTD itself is a significant predictor for costs $(p<0.001)$. We find deprivation category to be a main influencing factor for hospital costs.

Conclusion Our study confirms findings from other national research, and in addition shows interesting results for the effect that socio-economic status has on costs. This may be due to the restricted geographical area we are studying (Renfrew/Paisley), and leaves scope for further analysis using a sample representative for the whole of Scotland.

\section{GENDER DIFFERENCES IN ADMISSION TO CARE HOMES FOR OLDER PEOPLE: MAYBE MEN REALLY DO NOT CARE}

doi:10.1136/jech.2010.120956.64

${ }^{1} \mathrm{M}$ McCann, ${ }^{2} \mathrm{D}$ 0'Reilly. ${ }^{1}$ Institute of Child Care Research, Queen's University Belfast, UK; ${ }^{2}$ Centre for Public Health, Queen's University Belfast, UK

Objectives To offer reasons for the gender difference in care home admission risk.

Design A prospective longitudinal study collecting information from 2001 census returns, death registration, and health card registration information for a $28 \%$ sample of the Northern Ireland 